\title{
Utilisation des sources d'azote du lait par Lactococcus lactis
}

\author{
V Juillard, C Foucaud, M Desmazeaud, J Richard
}

Unité de recherches laitières, Inra, 75352 Jouy-en-Josas cedex, France

\begin{abstract}
Résumé - Les acides aminés libres du lait ne représentent qu'une faible part de l'azote aminé nécessaire à la croissance des lactocoques, puisqu'ils interviennent pour seulement $2 \%$ de la croissance totale. Ces bactéries, exigeantes d'un point de vue nutritionnel et auxotrophes pour certains acides aminés, doivent donc trouver une source d'azote complémentaire. L'utilisation des peptides du lait est conditionnée par leur transport à travers la membrane. L'étude du comportement de souches mutées pour un ou plusieurs systèmes de transport des peptides a montré que seuls quelques oligopeptides du lait sont utilisés. Ils interviennent pour $8 \%$ de la croissance totale. L'essentiel de la croissance des lactocoques dépend de l'utilisation des caséines. Cette utilisation requiert l'intervention de la protéase de paroi, qui libère un ensemble d'oligopeptides à partir des caséines. Seuls certains de ces oligopeptides pourront ensuite être transportés à l'intérieur de la cellule, via le système de transport des oligopeptides. Puisque tous les acides aminés sont représentés dans cet ensemble d'oligopeptides, ce système de transport suffit à l'approvisionnement de la cellule en acides aminés. Le système de tranport des oligopeptides joue donc un rôle primordial lors de la croissance dans le lait. En effet, les oligopeptides (qu'ils soient initialement présents dans le lait, ou qu'ils dérivent de la caséine) interviennent pour $98 \%$ de la croissance des lactocoques dans le lait.
\end{abstract}

\section{lactocoque / caséine / protéase / peptide / transport}

Summary - Utilization of nitrogen sources during growth of Lactococcus lactis in milk. Lactococci have very limited capacities of synthesizing amino acids and therefore must utilize exogenous nitrogen sources for optimal growth. Although lactococci possess several distinct amino acid transport systems, this nitrogen source plays a minor role during the growth process in milk. The use of the free amino acids of the milk accounts for only $2 \%$ of the total growth. Peptide utilization by lactococci mainly depends on their translocation abilities. Three different peptide transport systems have been identified in lactococci. However, only the oligopeptide transport system (Opp) is absolutely necessary for the use of the milk peptides as nitrogen source. Endogenous milk oligopeptides account for $8 \%$ of the total growth. Therefore, caseins represent the main source of amino acids. Casein utilization first requires a cell envelope-located proteinase, which releases only oligopeptides from caseins. Several of these oligopeptides are small enough to be translocated inside the cell via the Opp system. All the essential amino acids are present in these small oligopeptides, which therefore can supply the amino acid requirements of the cell. It is concluded that the Opp system plays a crucial role during the growth process in milk. It represents the main pathway for amino acid supply during growth in milk, since oligopeptides, whatever their origin (ie, initially present in milk or derived from caseins), account for $98 \%$ of the growth.

lactococci / casein / proteinase / peptide / transport 


\section{INTRODUCTION}

Les levains lactiques utilisés en industrie fromagère sont composés essentiellement, voire exclusivement, de lactocoques. D'un point de vue technologique, cette flore joue trois grands rôles. Elle est tout d'abord responsable de l'acidification du lait (fermentation lactique au cours de laquelle le lactose est dégradé en acide lactique). Elle est également à l'origine des modifications de flaveur, principalement du fait de son activité protéolytique : hydrolyse des caséines, formation de composés sapides (peptides amers) ou de précurseurs d'arôme (acides aminés). Enfin, elle permet de limiter le développement de germes indésirables, du fait de la production d'acide et d'éventuelles substances inhibitrices.

Pour pouvoir efficacement jouer ces différents rôles, la flore lactique doit atteindre un niveau de population important. Dans le lait, le principal facteur susceptible de limiter la croissance des lactocoques est leur nutrition azotée. En effet, ces bactéries sont exigeantes d'un point de vue nutritionnel, et auxotrophes pour au moins quatre acides aminés: His, Ile, Leu et Val (Chopin, 1993). Elles doivent donc trouver dans le lait l'azote assimilable qui leur est nécessaire. II y est présent sous trois formes : acides aminés libres, peptides et caséines. L'utilisation de ces sources d'azote par Lactococcus lactis varie naturellement selon leur nature.

\section{UTILISATION DES ACIDES AMINÉS LIBRES DU LAIT}

Les acides aminés peuvent être utilisés directement par le biais de transporteurs spécifiques. La spécificité et l'énergétique de ces différents systèmes de transport est connue depuis plusieurs années (voir revue de Konings et al, 1989), et ne seront pas détaillées ici.
Le lait contient de l'ordre de $85 \mathrm{mg} / \mathrm{l}$ d'acides aminés libres (Mills et Thomas, 1981 ; Juillard et al, 1995a). Néanmoins, certains y sont présents en faible quantité. En particulier, deux des acides aminés essentiels à la croissance de $L$ lactis, lle et Leu, sont présents à des concentrations nettement inférieures à celles requises pour atteindre une forte densité cellulaire (tableau I). En se basant sur les exigences de $L$ lactis subsp cremoris $E_{8}$ ou $A_{2}$, on peut estimer que la concentration du lait en leucine $(0,8 \mathrm{mg} / \mathrm{l})$ et en isoleucine $(0,6 \mathrm{mg} / \mathrm{l})$ devrait permettre d'atteindre une densité cellulaire de l'ordre de $3 \times 10^{7} \mathrm{ufc} / \mathrm{ml}$, soit 50 fois plus faible que celle normalement atteinte dans le lait.

La croissance de $L$ lactis subsp lactis CNRZ 1259 (variant non protéolytique de L lactis subsp lactis ML3) a été étudiée dans un milieu chimiquement défini contenant un mélange d'acides aminés comme seule source d'azote. La composition de ce mélange mimait exactement celle des acides aminés libres du lait. Le niveau cellulaire atteint en fin de culture était de l'ordre de $210^{7} \mathrm{ufc} / \mathrm{ml}$. Parallèlement, un mutant dérivé de L lactis subsp lactis ML3 ( $L$ lactis subsp lactis CFS 62), incapable d'utiliser les caséines et les peptides du lait (voir cidessous) a été obtenu (Foucaud et al, résultats non publiés). Sa croissance dans le lait ne repose donc que sur l'utilisation des acides aminés libres comme source d'azote. II y atteignait une densité cellulaire en fin de culture de l'ordre de $310^{7} \mathrm{ufc} / \mathrm{ml}$. Ces valeurs expérimentales sont donc comparables aux valeurs déduites des résultats de Mills et Thomas (1981). En effet, la croissance de $L$ lactis jusqu'à $310^{7} \mathrm{ufc} / \mathrm{ml}$ nécessiterait la synthèse d'approximativement $5 \mu \mathrm{g}$ de protéines bactériennes par $\mathrm{ml}$ de culture. Cette valeur correspond à la quantité d'acides aminés libres consommée au cours de la croissance d'un variant Prt de L lactis dans du lait (Juillard et al, 1995a). L'ensemble de ces résultats prouve donc 
Tableau I. Concentration de quelques acides aminés libres dans le lait et estimation des besoins pour la croissance de Lactococcus lactis subsp cremoris jusqu'en phase stationnaire.

Comparison of the concentration of some free amino acids in milk and the requirements for growth of Lactococcus lactis subsp cremoris to stationary phase.

Acide aminé

Concentration (mg/l)

\begin{tabular}{lccc} 
& Lait a & \multicolumn{2}{c}{ Requise pour la croissance $^{\text {b }}$} \\
\cline { 3 - 4 } & & \multicolumn{1}{c}{$A M_{2}$} & $E_{8}$ \\
\hline Isoleucine & $0,6 \pm 0,2$ & 12,5 & 13,2 \\
Leucine & $0,8 \pm 0,1$ & 21,5 & 22,8 \\
Valine & $2,1 \pm 0,7$ & 14,7 & 15,7 \\
Histidine & $4,8 \pm 0,3$ & 5,9 & 6,2 \\
Methionine & $0,7 \pm 0,2$ & 6,5 & 6,8 \\
Acide glutamique & $44,7 \pm 4,1$ & 40,1 & 44,8 \\
& & & 285 \\
Totaux & $84,0 \pm 4,8$ & 260 &
\end{tabular}

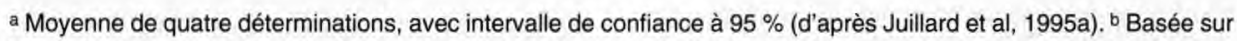
la composition en acides aminés des protéines de $L$ lactis subsp cremoris $\mathrm{AM}_{2}$ et $E_{8}$, respectivement (d'après Mills et Thomas, 1981).

a Mean of four determinations, with confidence limits at $\mathrm{P}=0.95$ (from Juillard et al, 1995a). ${ }^{b}$ Calculation was based on the amino acid composition of proteins of $L$ lactis subsp cremoris $A M_{2}$ and $E_{8}$, respectively (from Mills and Thomas, 1981).

que les acides aminés libres du lait ne jouent qu'un rôle mineur lors de la croissance de $L$ lactis dans le lait.

\section{UTILISATION DES PEPTIDES DU LAIT}

La connaissance des mécanismes d'utilisation des peptides par $L$ lactis a considérablement progressé ces 3 dernières années, bien que l'importance de cette source d'azote soit connue depuis longtemps (Mills et Thomas, 1981 ; Thomas et Mills, 1981). L'utilisation des peptides du lait, alliée à celle des acides aminés libres, permet d'atteindre des densités de population de l'ordre de $10^{8} \mathrm{ufc} / \mathrm{ml}$. Deux voies d'utilisation (non exclusives) peuvent être envisagées : soit un transport du peptide préalable à son hydrolyse par des pepti- dases intracellulaires, soit une hydrolyse par des peptidases exocellulaires libérant des acides aminés libres et des peptides plus courts, qui peuvent alors être transportés à l'intérieur de la cellule. L'existence d'une taille supérieure limite pour le transport des peptides, que l'on considérait jusqu'alors être de l'ordre de cinq à six acides aminés (Law, 1978), laissait supposer la coexistence des deux voies d'utilisation. Les travaux récents réalisés sur la caractérisation des peptidases de lactocoques, tant sur le plan biochimique que moléculaire, indiquent que toutes sont localisées à l'intérieur de la cellule (Tan et al, 1992 ; Kok et de Vos, 1994 ; Mierau et al, 1996). De ce fait, un transport des peptides préalablement à leur hydrolyse intracellulaire est l'enchaînement le plus vraisemblable.

Trois systèmes de transport des peptides ont été mis en évidence et caractéri- 
sés. Ils diffèrent par leur spécificité de substrat et leur énergétique (tableau II). Le système Opp transporte exclusivement des oligopeptides contenant au moins quatre acides aminés (Kunji et al, 1993 ; Tynkkynen et al, 1993). La taille supérieure limite n'est pas connue avec certitude. II a été établi que des oligopeptides de huit acides aminés sont transportés par le système Opp (Kunji et al, 1993), mais il semble que des peptides contenant jusqu'à 11 acides aminés puissent également être transportés (C Foucaud, résultats non publiés). La détermination de la taille supérieure limite pour le transport est rendue d'autant plus difficile que la spécificité de substrat du système Opp n'est pas connue. Le système Opp présente toutes les caractéristiques d'un ATPBinding-Cassette-transporteur : organisation du système en cinq sous-unités (une protéine de fixation des oligopeptides, OppA ; deux protéines hydrophobes transmembranaires, Opp B et $C$; deux protéines de fixation de l'ATP, Opp D et F), transport couplé à l'hydrolyse de l'ATP (Poolman et al, 1995). Les gènes codant le système Opp ont été clonés et séquencés (Tynkkynen et al, 1993). La disruption du gène oppA, codant la protéine de fixation OppA, a permis de montrer que le système Opp est le seul responsable du transport des oligo- peptides chez L lactis (Tynkkynen et al, 1993 ; Kunji et al, 1995).

Le système de transport DtpT a été le premier caractérisé chez $L$ lactis (Smid et al, 1989 ; Kunji et al, 1993). II transporte exclusivement des di- et tripeptides. Son activité est couplée à la force proton-motrice (translocation d'un peptide en symport avec un proton). Le système DtpT est codé par un seul gène, $d t p T$, qui a été cloné et séquencé (Hagting et al, 1994), Il code une protéine de 463 acides aminés, qui ne présente aucune homologie de séquence avec les autres systèmes de transport de peptides. La construction d'un mutant de délétion ( $L$ lactis AG300) a permis de préciser la spécificité de transport du système DtpT (tableau III).

L'existence d'un transport résiduel de certains di- et tripeptides par une souche mutée pour le système DtpT (tableau III) militait en faveur de l'existence d'un troisième système de transport des peptides chez $L$ lactis. II a récemment été mis en évidence et caractérisé (Foucaud et al, 1995). Ce troisième système, appelé DtpP, transporte préférentiellement des di- et tripeptides hydrophobes (contenant des acides aminés branchés). Toutefois, il existe entre les deux systèmes DtpT et DtpP un large recouvrement de spécificité. Le transport des di- et tripeptides via le système DtpP

Tableau II. Systèmes de transport des peptides caractérisés chez Lactococcus lactis. Peptide transport systems of Lactococcus lactis.

\begin{tabular}{|c|c|c|c|}
\hline Système & Spécificité & Énergétique & Référence \\
\hline Opp & $\begin{array}{l}\text { Oligopeptides } \\
\text { ( } 4 \text { à } 8 \text { résidus) }\end{array}$ & ATP-dépendant & $\begin{array}{c}\text { Kunji et al, } 1993 \\
\text { Tynkkynen et al, } 1993\end{array}$ \\
\hline DtpT & $\begin{array}{l}\text { Di- et tripeptides } \\
\text { hydrophiles }\end{array}$ & pmf-dépendant & $\begin{array}{l}\text { Smid et al, } 1989 \\
\text { Hagting et al, } 1994\end{array}$ \\
\hline DtpP & $\begin{array}{l}\text { Di- et tripeptides } \\
\text { hydrophobes }\end{array}$ & ATP-dépendant & Foucaud et al, 1995 \\
\hline
\end{tabular}


Tableau III. Transport de peptides par Lactococcus lactis (d'après Foucaud et al, 1995). Transport of peptides by Lactococcus lactis (from Foucaud et al, 1995).

\begin{tabular}{|c|c|c|c|c|c|}
\hline \multirow[t]{2}{*}{ Peptide } & \multicolumn{5}{|c|}{ Vitesse d'accumulation par L lactis ( $\mathrm{nmol} / \mathrm{min} / \mathrm{mg}$ protéine) } \\
\hline & $\begin{array}{c}M G 1363 \\
D t p T+, D t p P^{+} \\
O p p^{+}\end{array}$ & $\begin{array}{c}A G 300 \\
D t p T^{-}, D t p P^{+} \\
O p p^{+}\end{array}$ & $\begin{array}{c}C F 310 \\
D t p T^{-}, D t p P^{-} \\
\text {Opp }\end{array}$ & $\begin{array}{c}\text { VS772 } \\
D t p T^{+}, D t p P^{+} \\
\text {Opp }\end{array}$ & $\begin{array}{c}\text { CV4 } \\
D t p T-, D t p P^{+} \\
O p p^{-}\end{array}$ \\
\hline ProGly & 24 & $<0,1$ & $<0,1$ & 20 & $<0,1$ \\
\hline AlaGly & 67 & $<0,1$ & $<0,1$ & 61 & $<0,1$ \\
\hline LeuVal & 148 & 9 & $<0,1$ & 169 & 5 \\
\hline MetLeu & 50 & 21 & $<0,1$ & 60 & 24 \\
\hline MetMet & 190 & 29 & $<0,1$ & 159 & 32 \\
\hline TyrGly $_{2}$ PheLeu & 94 & 80 & 87 & $<0,1$ & $<0,1$ \\
\hline
\end{tabular}

est couplé à l'hydrolyse de l'ATP. L'inactivation des trois systèmes de transport DtpT, DtpP et Opp ( $L$ lactis CFS 62) abolit totalement le transport des peptides, ce qui suggère qu'il n'existe pas d'autre système de transport des peptides chez les lactocoques (C Foucaud, résultats non publiés).

L'obtention récente de mutants dérivés d'une souche dépourvue de protéase de paroi (souche Prt), et déficients pour l'un ou l'autre des systèmes de transport des peptides, a permis d'estimer le rôle joué par les peptides initialement présents dans le lait lors de la croissance (tableau IV). L'inactivation des systèmes de transport des di- et tripeptides (DtpT seul, ou DtpT et DtpP) n'affecte pas le niveau cellulaire en fin de culture. Toutefois, lorsque les deux systèmes de transport des di- et tripeptides sont inactivés, le taux de croissance de $L$ lactis CF310 serait ralenti (C Foucaud, résultats non publiés). Un ou plusieurs acides aminés seraient donc préférentiellement utilisés par $L$ lactis sous forme de di- ou tripeptides, bien qu'ils soient également présents dans le lait sous d'autres formes (vraisemblablement oligopeptides). Cette diminution du taux de croissance n'est pas observée lorsque seul le système DtpT est inactivé (Juillard et al, 1995a). Cela peut s'expliquer par le recouvrement de spécifi- cité qui existe entre les deux systèmes. Inversement, l'inactivation du système de transport des oligopeptides réduit d'un facteur 5 environ le niveau de population en fin de culture. Cela suggère donc que certains acides aminés essentiels à la croissance de $L$ lactis sont initialement présents dans le lait, principalement sous forme d'oligopeptides. Comme il n'existe pas de recouvrement de spécificité de substrat entre Opp d'une part, et DtpT et DtpP d'autre part, l'inactivation du système Opp ne peut être compensée par la présence de systèmes de transport des di- et tripeptides opérationnels. Le système de transport des oligopeptides joue donc un rôle primordial dans l'utilisation des peptides du lait. Enfin, la faible différence de niveau de population atteint par L lactis VS772 (Opp-, DtpT ${ }^{+}$, $\mathrm{DtpP}^{+}$) et $L$ lactis CFS62 (Opp ${ }^{-}$, DtpT-' $\left.\mathrm{DtpP}^{-}\right)$suggère un rôle quantitatif mineur des di- et tripeptides lors de la croissance d'une souche Prt.

L'analyse du contenu peptidique du lait avant et après croissance de ces différentes souches est en parfait accord avec ces résultats (Juillard et al, 1995a). Après la croissance de L lactis MG 1363 (DtpT+, $\left.\mathrm{DtpP}^{+}, \mathrm{Opp}^{+}\right)$, le lait n'est pas épuisé en peptides : seule une quinzaine de pics ne sont plus détectables. Le même résultat 
Tableau IV. Caractéristiques de croissance de souches de Lactococcus lactis mutées pour un ou plusieurs systèmes de transport des peptides (d'après Juillard et al, 1995a ; Foucaud et al, résultats non publiés).

Growth features of Lactococcus lactis strains with inactivated peptide transport systems (from Juillard et al, 1995a; Foucaud et al, unpublished results).

L lactis subsp lactis a $\quad$ Phénotype Niveau de population en fin de culture
dans du lait (ufc/ml) ${ }^{\mathrm{b}}$

\begin{tabular}{|c|c|c|}
\hline MG 1363 & $\mathrm{DtpT}^{+}, \mathrm{DtpP}+, \mathrm{Opp}^{+}$ & $(1,6 \pm 0,3) 10^{8}$ \\
\hline AG 300 & DtpT-, DtpP+, Opp+ & $(1,5 \pm 0,5) 10^{8}$ \\
\hline CF 310 & DtpT-, DtpP-, Opp+ & $(1,1 \pm 0,3) 10^{8}$ \\
\hline VS 772 & $\mathrm{DtpT}^{+}, \mathrm{DtpP}+, \mathrm{Opp}-$ & $(3,8 \pm 0,5) 10$ \\
\hline CV 4 & DtpT- $\mathrm{DtpP}^{+}, \mathrm{Opp}^{-}$ & $(3,7 \pm 0,7) 10^{7}$ \\
\hline CFS 62 & $\mathrm{DtoT}^{-}$DtoP- Ono- & $(2.7 \pm 0.2) 10^{7}$ \\
\hline
\end{tabular}

\begin{abstract}
a Les phénotypes DipT-et Opp- ont respectivement été obtenus par délétion du gène dtpT (Hagting et al, 1994) ou par disruption du gène oppA (Tynkkynen et al, 1993). Les souches CF 310 et CFS 62 sont des mutants spontanés de résistance au peptide toxique Phe-ßchloro-Ala, obtenus à partir respectivement de L lactis AG 300 (Foucaud et al, 1995) ou de L lactis CV4 (Foucaud et al, résultats non publiés). 'b Moyenne de quatre déterminations, avec intervalle de confiance à $95 \%$. Niveau d'ensemencement du lait : $10^{6} \mathrm{ufc} / \mathrm{ml}$.

a DtpT- and Opp-phenotypes resulted from the deletion of the dtp T gene (Hagting et al, 1994) and from the disruption of the oppA gene (Tynkkynen et al, 1993), respectively. Both L lactis CF310 and CFS62 strains were selected for resistance towards the toxic peptide Phe-Bchloro-Ala from L lactis AG300 (Foucaud et al, 1995) and L lactis CV4 (Foucaud et al, unpublished results), respectively, ${ }^{\circ}$ Mean of four determinations, with confidence limits at $\mathrm{P}=0.95$. Inoculation level of the milk was $10^{6} \mathrm{cfu} / \mathrm{ml}$.
\end{abstract}

était observé après la croissance de $L$ lactis AG300 (DtpT-, DtpP+, Opp ${ }^{+}$). Inversement, après la croissance de L lactis VS 772 $\left(\mathrm{DtpT}^{+}, \mathrm{DtpP}^{+}, \mathrm{Opp}^{-}\right)$, l'aire de deux pics seulement avait diminué. Les peptides du lait qui sont utilisés pendant la croissance sont donc des oligopeptides. II convient maintenant de les identifier, et d'expliquer pourquoi les autres peptides du lait ne sont pas utilisés (problème de spécificité par rapport à la taille de ces peptides et/ou par rapport à leur séquence...).

\section{UTILISATION DES CASÉINES}

Les caséines du lait représentent la principale source d'azote pour les lactocoques: $90 \%$ de la croissance leur est imputable. Leur utilisation met en oeuvre un système complexe, qui fait intervenir plusieurs enzymes agissant séquentiellement. La protéase de paroi réalise la première étape du processus de dégradation des caséines du lait. Sa présence est nécessaire au développement de $L$ lactis jusqu'à de fortes densités cellulaires (Thomas et Mills, 1981).

Les caractéristiques biochimiques et génétiques des protéases de paroi de $L$ lactis ont été étudiées dans le détail et ne seront pas développées ici. Le lecteur est invité à se référer aux revues bibliographiques très complètes qui ont été publiées à ce sujet (Pritchard et Coolbear, 1993 ; Tan et al, 1993 ; Kok et de Vos, 1994). D'un point de vue physiologique, on distingue deux principales catégories de protéases, différant par leur spécificité d'action sur les caséines du lait (tableau V). En particulier, la spécificité (et la vitesse) d'hydrolyse de la caséine $\beta$ par les protéases de type $P_{1}$ et

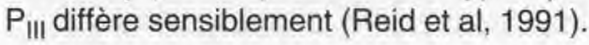


La caséine $\beta$ représente le substrat le mieux hydrolysé par les protéases de paroi. C'est également la caséine utilisée préférentiellement lors de la croissance de $L$ lactis (Exterkate et de Veer, 1987). De ce fait, de nombreuses études ont porté sur l'identification des peptides liberés après hydrolyse de la caséine $\beta$ par une protéase de paroi purifiée (Visser et al, 1988, 1991 ; Monnet et al, 1989 ; Reid et al, 1991). Du fait de contraintes méthodologiques (nécessité de purifier les peptides avant de les identifier), seuls les peptides les plus abondants avaient été analysés. Une quarantaine de peptides avaient ainsi été identifiés, comportant de trois à 25 acides aminés. Pour pouvoir être utilisés par $L$ lactis, ces peptides doivent contenir au plus huit résidus. Très peu de peptides de huit résidus ou moins avaient été identifiés, et ils ne contenaient pas tous les acides aminés indispensables à la croissance de $L$ lactis. Puisque toutes les peptidases sont localisées à l'intérieur de la cellule, la croissance des lactocoques dans des milieux à base de caséines ne peut réellement s'expliquer que si l'hydrolyse des caséines libère un plus grand nombre de peptides de huit résidus ou moins.
Une analyse fine de la dégradation de la caséine $\beta$ par la protéase de paroi de $L$ lactis Wg2 a donc été développée. Le couplage d'un spectromètre de masse à un chromatographe liquide haute performance (HPLC) a permis d'identifier l'ensemble des peptides libérés (Juillard et al, 1995b). En effet, ce montage (LC/MS) permet de déterminer le poids moléculaire de chaque peptide, même si la séparation durant la chromatographie est imparfaite (cas de peptides co-élués), rendant superflue l'étape de purification des peptides à identifier. Une première série d'injections de l'hydrolysat de caséine était réalisée, dans des conditions favorisant la formation d'ions multi-chargés. Cela permettait de déterminer la masse moléculaire de chacun des peptides constituant le mélange. Dans la plupart des cas, une masse déterminée pouvait correspondre à différents fragments de la molécule de caséine $\beta$. Une seconde série d'injections de l'hydrolysat a alors été réalisée, dans des conditions expérimentales permettant la fragmentation des peptides constituant le mélange (collision par dissociation induite). L'analyse des ions résultant de la dissociation d'un peptide permettait son identification sans aucun doute possible. Cette

Tableau V. Spécificité de substrat des protéases de paroi de Lactococcus lactis. Substrate specificity of the cell envelope-located proteinase of Lactococcus lactis.

Type de protéase

$P_{I} \quad P_{I I I}$

Substrats préférentiels

Souche type

Nombre de peptides libérés après hydrolyse de la caséine $\beta$
Caséine $\beta$

( $\kappa$ dans une moindre mesure)

L lactis Wg2

39 a
Caséines $\beta, \kappa, \alpha_{\mathrm{s}}$

L lactis SK11

$32^{a}$

\footnotetext{
a D'après Tan et al, 1993

a From Tan et al, 1993.
} 
approche a prouvé que l'hydrolyse de la caséine $\beta$ par une protéase de paroi génère un nombre de peptides beaucoup plus important que ce que l'on pensait jusqu'alors. Les peptides libérés contiennent de quatre à 30 acides aminés (fig 1). II convient de souligner qu'aucun dipeptide ou tripeptide n'a pu être identifié, et que seul un acide aminé libre (Phe) était libéré.

Parmi cet ensemble de peptides, 18 contiennent moins de neuf acides aminés et sont donc susceptibles d'être transportés à l'intérieur de la cellule via le système Opp (fig 2). Chacun de ces 18 peptides contient au moins un acide aminé essentiel à la croissance. De plus, tous les acides aminés constitutifs de la caséine $\beta$ (hormis la phosphosérine) sont présents dans cet ensemble de peptides, qui devrait donc théoriquement suffire à la croissance de $L$ lactis.

Le comportement de souches $\mathrm{Prt}^{+}$de lactocoques, mutées pour l'un ou l'autre des systèmes de transport, et incubées avec de la caséine $\beta$ comme seul substrat, est en parfait accord avec cette hypothèse (Kunji et al, 1995). L'utilisation des peptides exocellulaires par les différentes souches était estimée par l'augmentation du pool interne d'acides aminés. En effet, l'équipement peptidasique intracellulaire des lactocoques est tel qu'il n'est pas possible de mesurer l'accumulation de peptides, trop rapidement hydrolysés. Tous les acides aminés constitutifs de la caséine $\beta$ sont retrouvés à l'intérieur de la cellule, dès lors que le système de transport des oligopeptides est fonctionnel. À l'inverse, aucune accumulation significative n'est détectée en l'absence de transport des oligopeptides. Comme on pouvait le supposer, la mutation du système DtpT n'a aucun effet sur l'utilisation des peptides issus de la dégradation de la caséine. Enfin, la croissance dans du lait de ces différents mutants corrobore les résultats des expériences de transport. Le niveau cellu-

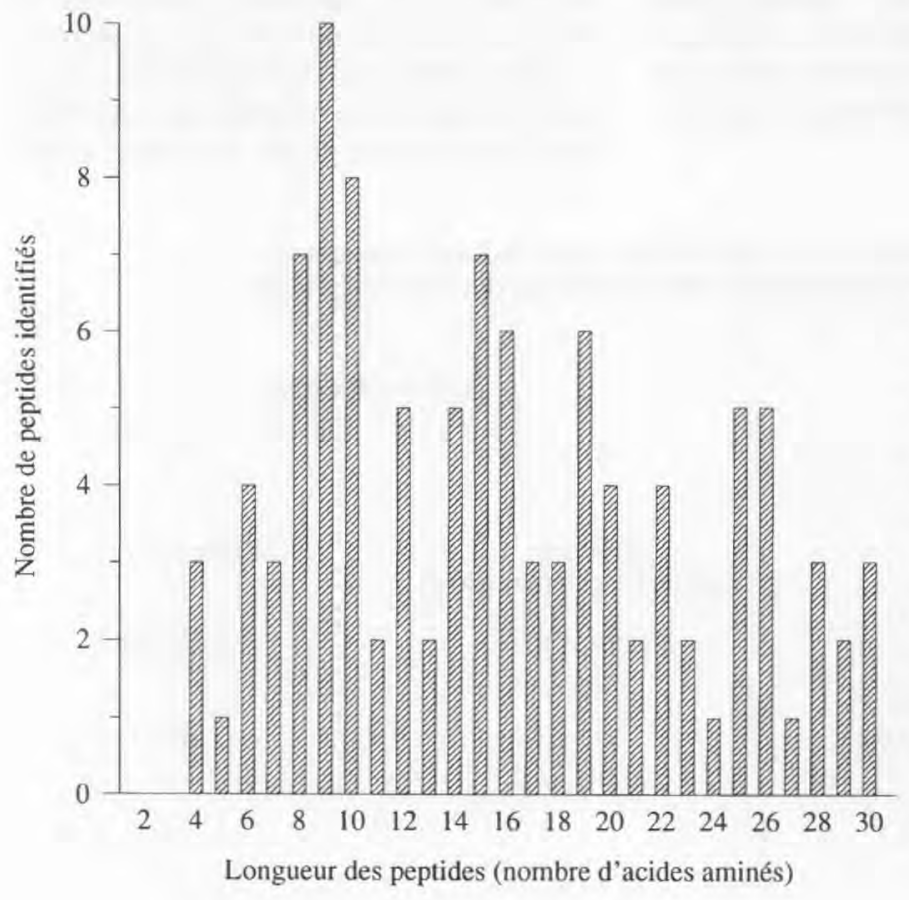

Fig 1. Répartition des peptides issus de la dégradation de la caséine $\beta$ par la protéase de paroi de Lactococcus lactis subsp cremoris Wg2 en fonction de leur taille (d'après Juillard et al, 1995b).

Size distribution of peptides released by the action of purified proteinase from Lactococcus lactis subsp cremoris $W g 2$ on $\beta$-casein (from Juillard et al, 1995b). 
Fig 2. Localisation, sur la séquence de la caséine $\beta$, des peptides de moins de neuf acides aminés résultant de I'hydrolyse par la protéase de paroi de Lactococcus lactis subsp cremoris Wg2 (d'après Juillard et al, 1995b).

Localization of peptides containing eight amino acids or less released by the action of purified proteinase from Lactococcus lactis subsp cremoris $W g 2$ on $\beta$-casein (from Juillard et al, 1995b).

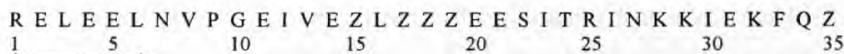

E E Q Q Q T E D E L Q D K I H P F A T Q S L V Y P F G P I P N L L 40 45

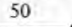

55

60

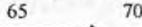

PQN I P P L T Q T P V V V P P F Q P E V M G V S K V K E A M A P K 75 85 90 95 100 105

H K E M P P K Y P V Q P T E Q S L T L T D V E L H L P L P L L

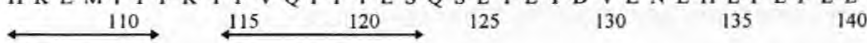

Q S W M H Q P H P L P P T V F P P Q S V L S L S Q S K V L P V P Q

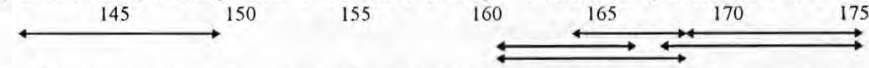

K A V P Y P Q R D M P I Q A F L L Y Q Q P V L G P V R G P F P I I V $\stackrel{180}{\longleftrightarrow} \stackrel{185}{\longleftrightarrow}$ laire atteint par une souche $\mathrm{Prt}^{+}$incapable de transloquer les oligopeptides représente moins de $5 \%$ de celui atteint par la souche sauvage (Kunji et al, 1995).

\section{LIMITES DU SYSTÈME PROTÉOLYTIQUE}

Si la présence du système protéolytique est indispensable à la croissance de $L$ lactis dans le lait jusqu'à de fortes densités cellulaires, son fonctionnement présente néanmoins des limitations. En effet, la croissance d'une souche $\mathrm{Prt}^{+}$de lactocoque dans du lait se déroule suivant deux phases exponentielles distinctes (fig 3 ). Cette seconde phase de croissance n'est pas détectable lors de la culture d'un variant Prt, puisqu'elle correspond à l'utilisation des caséines du lait comme source d'azote (Juillard et Richard, 1991). La vitesse de croissance en seconde phase est plus lente que lors de la première phase, qui correspond à l'utilisation des acides aminés libres et des oligopeptides endogènes du lait.

Pendant la seconde phase de croissance, il y a accumulation d'acides aminés libres et de peptides dans le lait (Juillard et

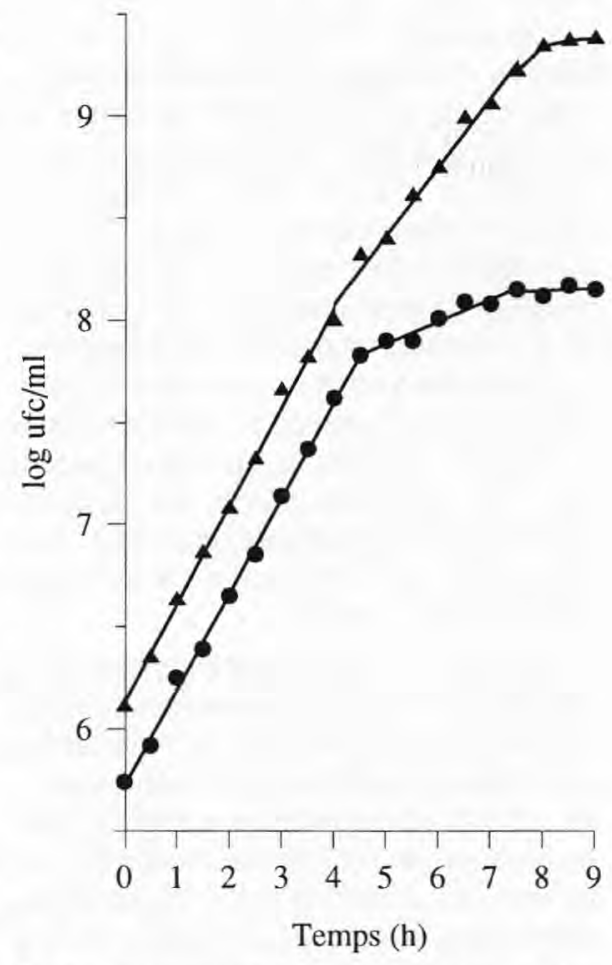

Fig 3. Croissance de Lactococcus lactis subsp lactis ML3 dans du lait. $\boldsymbol{\Delta}$ : souche $\mathrm{Prt}^{+} ; \bullet$ : souche Prt-.

Growth of Lactococcus lactis subsp lactis ML3 in milk. $\mathbf{A}$ : Prt+ strain; : Prt strain. 
al, 1995a). Ces substrats azotés non protéiques ne permettent pas la croissance d'un variant Prt'. Cela signifie donc que seule une petite partie des produits de protéolyse est utilisable pour la croissance, ce qui est en parfait accord avec les résultats d'identification de produits de protéolyse obtenus in vitro. Cela signifie également que cette fraction utilisable n'est pas accumulée dans le lait au cours de la croissance $d^{\prime}$ 'une souche $\mathrm{Prt}^{+}$. Cela sous-entend donc que la vitesse de croissance à partir des caséines du lait est limitée par la vitesse de dégradation de ces substrats, et non par la vitesse de transport des peptides résultant de l'hydrolyse (sans quoi il y aurait accumulation dans le lait de substrats utilisables pour la croissance d'un variant Prt).

L'étude de la croissance dans le lait de souches de lactocoque surproduisant la protéase de paroi n'a pas permis de mettre en évidence une stimulation significative de la vitesse de multiplication cellulaire en seconde phase exponentielle, du fait d'un trop faible facteur de surproduction de la protéase de paroi (inférieur à 2). L'ajout au lait d'une solution contenant une protéase de paroi purifiée a permis de stimuler de $30 \%$ la vitesse de croissance en seconde phase (S Helinck, résultats non publiés). Cependant, une forte diminution du taux de croissance était rapidement observée. Les causes de cette inhibition secondaire sont actuellement à l'étude.

Quoi qu'il en soit, cette limitation de la vitesse de croissance par la vitesse de protéolyse a des répercussions importantes sur le plan technologique. Contrairement à ce qui était communément admis, la «prématuration" du lait par une souche $\mathrm{Prt}^{+}$ne permet pas d'enrichir le lait en substrats azotés non protéiques utilisables pour la croissance. Cela se traduit par une moins bonne croissance de la souche cultivée en second, et donc un retard à la coagulation (Juillard et Richard, 1991). De même, cette limitation est en contradiction avec l'hypo-

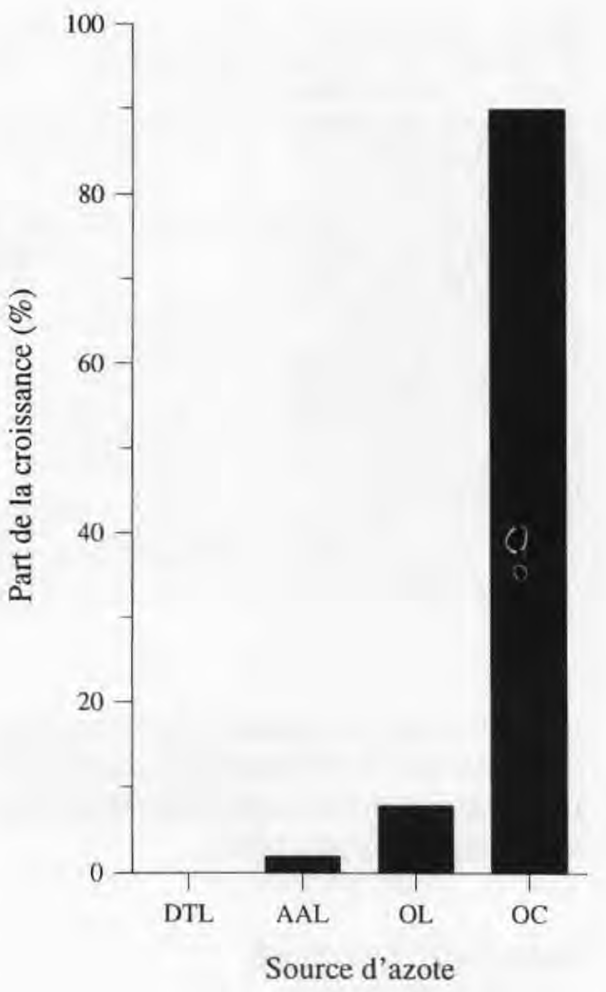

Fig 4. Incidence des différentes sources d'azote du lait sur la croissance de Lactococcus lactis (d'après Juillard et al, 1995a). DTL : di- et tripeptides initialement présents dans le lait ; AAL : acides aminés libre du lait ; OL : oligopeptides initialement présents dans le lait ; OC : oligopeptides dérivés de l'hydrolyse des caséines par la protéase de paroi.

Contribution of different nitrogen sources to growth of Lactococcus lactis in milk (from Juillard et al, 1995a). DTL: di- and tripeptides initially present in milk; AAL: free amino acids initially present in milk; OL: oligopeptides initially present in milk; OC: oligopeptides released from caseins by the action of the cell envelope-located proteinase.

thèse communément admise d'une relation de commensalisme existant entre une souche $\mathrm{Prt}^{+}$et un variant $\mathrm{Prt}$ cultivés en association dans du lait. Si le variant $\mathrm{Prt}^{-}$ est bien stimulé, la souche parentale est partiellement inhibée, du fait d'une compétition entre les deux souches pour les pro- 


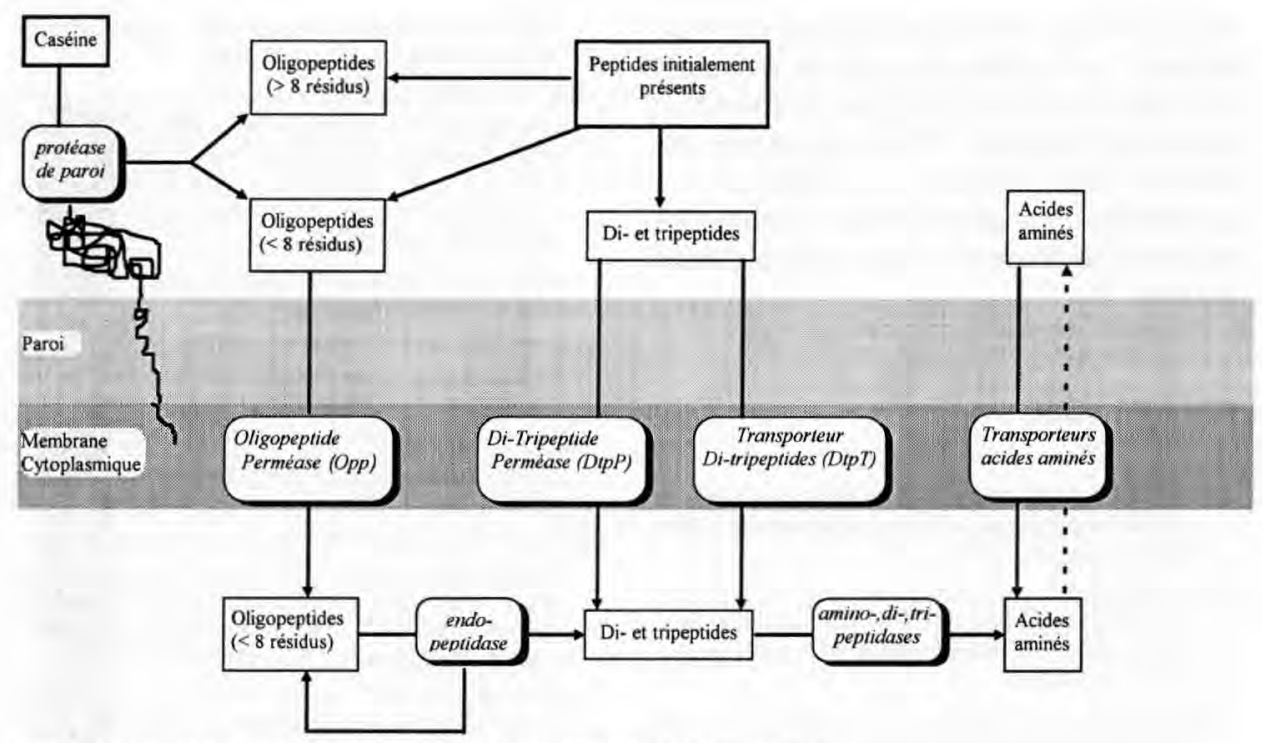

Fig 5. Utilisation des sources d'azote du lait par Lactococcus lactis.

Utilization of milk nitrogen sources by Lactococcus lactis.

duits de protéolyse (Juillard et Richard, 1994). Plus la proportion de variants Prt dans le mélange est importante, plus la compétition est marquée, et plus l'inhibition de la souche parentale est sensible. En particulier, au delà d'un certain pourcentage de variants $\mathrm{Prt}^{-}$dans le mélange, on observe un retard à l'acidification du lait.

\section{CONCLUSIONS}

L'expression des propriétés fermentaires des lactocoques à des fins industrielles requiert une croissance rapide, jusqu'à de fortes densités cellulaires. Dans le lait, cet impératif technologique dépend en grande partie de la nutrition azotée de ces microorganismes. Les mécanismes régissant l'approvisionnement des lactocoques en acides aminés sont aujourd'hui mieux compris. Ils font intervenir un ensemble d'enzymes et de transporteurs, parmi lesquels deux ont une importance capitale. La protéase de paroi permet l'utilisation des caséines comme source d'azote. Elle libère un ensemble d'oligopeptides, dont seuls quelques-uns sont utilisés comme source d'acides aminés. Pour un certain nombre de souches, la vitesse de production de ces oligopeptides limite la vitesse de croissance. Toutefois, cette vitesse semble correspondre à un équilibre biologique, puisqu'une forte augmentation de la vitesse de dégradation des caséines (réalisée par l'apport exogène au lait de protéase purifiée) induit rapidement un effet inhibiteur, dont les origines restent à déterminer. L'autre système indispensable à l'utilisation des sources d'azote du lait est le transporteur des oligopeptides. En effet, les oligopeptides, qu'ils dérivent de la caséine ou qu'ils soient initialement présents dans le lait, représentent la principale source d'acides aminés pour les lactocoques, puisque $98 \%$ de la croissance des lactocoques leur est imputable (fig 4).

Si le schéma d'utilisation des sources d'azote du lait est aujourd'hui mieux compris 
(fig 5), il reste néanmoins plusieurs points à éclaircir, au nombre desquels le rôle exact joué par les deux systèmes de transport des di- et tripeptides, et la spécificité du système de transport des oligopeptides, dont la connaissance permettra de comprendre pourquoi seulement certains oligopeptides sont utilisés.

\section{RÉFÉRENCES}

Chopin A (1993) Organization and regulation of genes for amino acid biosynthesis in lactic acid bacteria. FEMS Microbiol Rev 12, 21-38

Exterkate FA, de Veer GJCM (1987) Optimal growth of Streptococcus cremoris HP in milk is related to $\beta$ and $\mathrm{k}$-casein degradation. Appl Microbiol Biotech$n o l 25,471-475$

Foucaud C, Kunji ERS, Hagting A, Richard J, Konings WN, Desmazeaud M, Poolman B (1995) Specificity of peptide transport systems in Lactococcus lactis: evidence for a third system which transports hydrophobic di- and tripeptides. J Bacteriol 177, 4652-4657

Hagting A, Kunji ERS, Leenhouts KJ, Poolman B, Konings WN (1994) The di- and tripeptide transport protein of Lactococcus lactis. A new type of bacterial peptide transporter. J Blol Chem 269, 11391-11399

Juillard V, Richard J (1991) Indirect interaction in milk between proteolytic and isogenic non-proteolytic strains of Lactococcus lactis. II. Effect of pre-culturing milk by a proteolytic strain. Lait 71, 55-64

Juillard V, Richard J (1994) Mixed culture in milk of a proteinase-positive and a proteinase-negative variant of Lactococcus lactis subsp lactis: influence of initial percentage of proteinase-positive cells on the growth parameters of each strain and on the rate of acidification. Lait 74, 3-12

Juillard V, Le Bars D, Kunji ERS, Konings WN, Gripon JC, Richard J (1995a) Oligopeptides are the main source of nitrogen for Lactococcus lactis during growth in milk. Appl Environ Microbiol61, 3024-3030

Juillard V, Laan $\mathrm{H}$, Kunji ERS, Jeronimus-Stratingh MJ, Bruins AP, Konings WN (1995b) The extracellular $P_{1}$-type proteinase of Lactococcus lactis hydrolyses $\beta$-casein into more than one hundred different oligopeptides. J Bacteriol 177, 3472-3478

Kok J, de Vos WM (1994) The proteolytic system of lactic acid bacteria. In: Genetics and biotechnology of lactic acid bacteria (Gasson MJ, de Vos WM, eds) Blackie Academic and Professional, Glasgow

Konings WN, Poolman B, Driessen AJM (1989) Bioenergetics and solute transport in lactococci. Crit Rev Microbiol 16, 419-476

Kunji ERS, Smid EJ, Plapp R, Poolman B, Konings WN (1993) Di-tripeptides and oligopeptides are taken up via distinct transport mechanisms in Lactococcus lactis. J Bacteriol 175, 2052-2059

Kunji ERS, Hagting A, de Vries CJ, Juillard V, Haandrikman AJ, Poolman B, Konings WN (1995) Transport of $\beta$-casein derived peptides by the oligopeptide transport system is a crucial step in the proteolytic pathway of Lactococcus lactis. $J$ Biol Chem 270, 1569-1574

Law BA (1978) Peptide utilization by group $N$ streptococci. $J$ Gen Microbial 105, 113-118

Mills OE, Thomas TD (1981) Nitrogen sources for growth of lactic streptococci in milk. NZJ Dairy Scf Technol $16,43-55$

Mierau I, Kunji ERS, Venema G, Poolman B, Kok J (1996) Peptidases and growth of Lactococcus lactis in milk. Lait 76 "dans ce même numéra"

Monnet V, Bockelmann W, Gripon JC, Teuber M (1989) Comparison of cell wall proteinase from Lactococcus lactis subsp cremoris AC1 and Lactococcus lactis subsp lactis NCDO 763. II. Specifiticy towards $\beta$ casein. Appl Microbiol Biotechnol 31, 112-118

Poolman B, Kunji ERS, Hagting A, Juillard V, Konings WN (1995) The proteolytic pathway of Lactococcus lactis. J Appl Bacteriol, Symp Suppl 79, 65S-75S

Pritchard GG, Coolbear T (1993) The physiology and biochemistry of the proteolytic system in lactic acid bacteria. FEMS Microbiol Rev 12, 172-206

Reid JR, Huat NK, Moore CH, Coolbear T, Pritchard GG (1991) Comparison of bovine $\beta$-casein hydrolysis by $P_{1}$ and $P_{\text {III }}$ type proteinase from Lactococcus lactis subsp cremoris. Appl Microbiol Biotechnol 36, 344-351

Smid EJ, Driessen AJM, Konings WN (1989) Mechanism and energetics of dipeptide transport in membrane vesicles of Lactococcus lactis, J Bacteriol 171. 292-298

Tan PST, Chapot-Chartier MP, Pos KM, Rousseau M, Bocquien CY, Gripon JC, Konings WN (1992) Localization of peptidases in lactococci. Appl Environ Microbiol 58, 285-290

Tan PST, Poolman B, Konings WN (1993) Proteolytic enzymes of Lactococcus lactis. J Dairy Res 60, 269 286

Thomas TD, Mills OE (1981) Proteolytic enzymes of starter bacteria. Neth Milk Dairy J 35, 255-273

Tynkkynen S, Buist G, Kunji ERS, Kok J, Poolman B, Venema G, Haandrikman AJ (1993) Genetic and biochemical characterization of the oligopeptide transport system of Lactococcus lactis. J Bacteriol $175,7523-7532$

Visser S, Slangen CJ, Exterkate FA, de Veer GJCM (1988) Action of a cell wall proteinase $\left(P_{i}\right)$ from Streptococcus cremoris HP on bovine $\beta$-casein. Appl Microbiol Biotechnol 29, 61-66

Visser S, Robben AJPM, Slangen CJ (1991) Specificity of a cell-envelope-located proteinase ( $P_{\| 1}$-type) from Lactococcus lactis subsp cremoris AM1 in its action on bovine $\beta$-casein. Appl Microbiol Biotechnol 35 , $477-483$ 